\title{
Test characteristics of two rapid antigen detection tests (SD FK50 and SD FK60) for the diagnosis of malaria in returned travellers Mirna Van der Palen ${ }^{1}$, Philippe Gillet ${ }^{2}$, Emmanuel Bottieau ${ }^{2}$, Lieselotte Cnops ${ }^{2}$, Marjan Van Esbroeck ${ }^{2}$ and Jan Jacobs*1,2
}

\author{
Address: ${ }^{1}$ Faculty of Health, Medicine and Life Sciences (FHML), Maastricht, The Netherlands and ${ }^{2}$ Department of Clinical Medicine, Institute of \\ Tropical Medicine (ITM), Antwerp, Belgium \\ Email: Mirna Van der Palen - mirna_palen@yahoo.com; Philippe Gillet - pgillet@itg.be; Emmanuel Bottieau - ebottieau@itg.be; \\ Lieselotte Cnops - lcnops@itg.be; Marjan Van Esbroeck - mvesbroeck@itg.be; Jan Jacobs* - jjacobs@itg.be \\ * Corresponding author
}

Published: 5 May 2009

Malaria Journal 2009, 8:90 doi:10.1 186/1475-2875-8-90

This article is available from: http://www.malariajournal.com/content/8/I/90

(C) 2009 Palen et al; licensee BioMed Central Ltd.

This is an Open Access article distributed under the terms of the Creative Commons Attribution License (http://creativecommons.org/licenses/by/2.0), which permits unrestricted use, distribution, and reproduction in any medium, provided the original work is properly cited.
Received: 23 November 2008

Accepted: 5 May 2009

\begin{abstract}
Background: Two malaria rapid diagnostic tests were evaluated in a travel clinic setting: the SD FK50 Malaria Ag Plasmodium falciparum test (a two-band test) and the SD FK60 Malaria Ag P. falciparum/Pan test (a three-band test).

Methods: A panel of stored whole blood samples $(n=452$ and $n=614$ for FK50 and FK60, respectively) from returned travellers was used. The reference method was microscopy with PCR in case of discordant results.

Results: For both tests, overall sensitivity for the detection of $P$. falciparum was $93.5 \%$, reaching $97.6 \%$ and $100 \%$ at parasite densities above 100 and $\mathrm{I}, 000 / \mu \mathrm{l}$ respectively. Overall sensitivities for Plasmodium vivax, Plasmodium ovale and Plasmodium malariae for the FK60 test were 87.5\%, 76.3\% and $45.2 \%$, but they reached $92.6 \%$ and $90.5 \%$ for $P$. vivax and $P$. ovale at parasite densities above $500 / \mu \mathrm{l}$. Specificities were above $95 \%$ for all species and both tests when corrected by PCR, with visible histidine-rich protein-2 lines for $P$. malariae $(n=3)$ and $P$. vivax and $P$. ovale (I sample each). Line intensities were reproducible and correlated to parasite densities. The FK 60 tests provided clues to estimate parasite densities for $P$. falciparum below or above $1,000 / \mu \mathrm{l}$.
\end{abstract}

Conclusion: Both the FK50 and FK60 performed well for the diagnosis of $P$. falciparum in the present setting, and the FK60 for the diagnosis of $P$. vivax and $P$. ovale at parasite densities $>500 / \mu$. The potential use of the FK60 as a semi-quantitative estimation of parasite density needs to be further explored.

\section{Background}

Microscopic diagnosis of malaria requires considerable training and experience. Most diagnostic laboratories in non-endemic countries lack sufficient samples to enable building-up and maintenance of microscopic expertise. In addition, many returned travellers suspected of malaria present outside office hours, when expert microscopy may not be at hand $[1,2]$. Rapid diagnostic tests (RDTs) offer a simple and rapid complement to microscopic malaria diagnosis. The earlier two-band tests were designed to detect Plasmodium falciparum. They display a control line and a test line, which targets either histidine-rich protein- 
2 (HRP-2) or P. falciparum-specific parasite lactate dehydrogenase $(\mathrm{pLDH})$. Newer generation three-band tests display a control line and two test lines, one for detection of $P$. falciparum-specific antigen and another for detection of antigens common to the four species, such as pan-Plasmodium-specific parasite lactate dehydrogenase or aldolase. Simplified "one-step" malaria RDTs have been marketed. Unlike their predecessors, the one-step RDTs only require one or two manipulations, i.e. application of blood and a running buffer. It is to be expected that they will increase performance by laboratory and clinical staff who are using the RDTs on an incidental base. Indeed, multistep RDTs have been demonstrated to require considerable training to reach optimal sensitivity [2]. Many brands are marketed, but published reports are only available for a small number of them [3-6]. The World Health Organization, through the Regional Office for the Western Pacific, lists a number of malaria RDTs, which are produced in compliance with ISO 13485:2003 [7].

The SD FK50 Malaria Ag P. falciparum test (Standard Diagnostics, Hagal-Dong, Korea) and the SD FK60 Malaria Ag $P$. falciparum/Pan (Standard Diagnostics) are one-step malaria diagnostic tests in a cassette format, in a two- and three-band design, respectively. In this study, their performance was assessed when challenged with a collection of stored blood samples of returned travellers in a reference centre. For convenience, these tests will be referred to as FK50 and FK60, respectively.

\section{Methods}

\section{Study design}

Both kits were retrospectively evaluated in a reference laboratory on a panel of stored blood samples obtained in returned travellers suspected of malaria. The reference method was microscopy, performed at presentation of the patient. All discordant results were subsequently analysed by PCR, and test characteristics were recalculated according to the PCR-corrected results.

\section{Patients and Materials}

EDTA-blood samples from patients attending the outpatient clinic of the Institute of Tropical Medicine (ITM), Antwerp, Belgium, or those that were sent by Belgian laboratories to ITM for confirmation in the scope of the national reference function were used. Patients included European travellers returning from malaria-endemic areas and, to a lesser extent, natives of endemic countries returning from visiting friends and relatives. The samples were submitted as part of the diagnostic protocol for suspected malaria. Samples had been collected from January 1996 to October 2007 and had been stored at $-70^{\circ} \mathrm{C}$. Diagnosis was based on standard microscopy. Among these samples, a panel was selected based on relevant representation of the four malaria species ( $P$. falciparum, Plasmodium vivax, Plasmodium ovale and Plasmodium malariae) and different parasite densities. For the FK60, additional samples of non-falciparum species were included. Samples without malaria parasites (negative samples) were collected prospectively during the period of September and October 2007 from returned travellers attending the outpatient clinic of ITM and for whom a thick film, requested as part of work-up of suspected malaria, did not show any malaria parasites.

\section{Reference method}

Diagnosis of malaria, species identification and determination of parasite density were done by microscopy. According to standard practice at the ITM, thick and thin blood films were prepared, stained with Giemsa ( $\mathrm{pH} 8.0)$ and examined by light microscopy using a $\times 500$ magnification. An examination of 15 minutes for a thick film, with a minimum of 200 fields read, was performed before the blood film was reported negative. Parasite densities were estimated by counting asexual parasites against 200 white blood cells (WBC) in thick blood films, converting this number to parasites/ $\mu \mathrm{l}$ using the actual WBC count or, when this was not available, the standard 8,000 WBC/ $\mu \mathrm{l}$ value [4]. Parasite densities are further in this text expressed as counts (of asexual parasites)/ $\mu$ l (of whole blood).

\section{Test platforms}

The FK50 is a two-band RDT targeting HRP-2 antigen. Results are expressed as positive or negative for $P$. falciparum. The FK60 is a three-band test targeting HRP-2 and $\mathrm{pLDH}$. The presence of a HRP-2 line together with a pLDH line indicates an infection with $P$. falciparum or a mixed infection with $P$. falciparum and one or more of the other Plasmodium species. The presence of a unique HRP-2 line refers to an infection with $P$. falciparum, whereas a unique pLDH line indicates infection with one or more of the other Plasmodium species. Both assays are lateral flow immunochromatographic antigen detection tests in a cassette format.

\section{Test procedure}

Tests were performed according to the instructions of the manufacturer, except that samples $(5 \mu \mathrm{l})$ were loaded with a transfer pipette (Finnpipette, Helsinki, Finland) instead of the plastic tube supplied by the manufacturer and that a scoring system was used to assess the intensity of the test lines. In cases for which the control line did not appear, the results were interpreted as invalid and the tests were repeated. In order to score test line intensities, the scoring system of Bell and co-workers [8]was used and five categories were defined: None (no line visible), Faint (barely visible line), Weak (paler than the control line), Medium (equal to the control line) or Strong (stronger than the control line). To assure timely readings, tests were carried out in time-controlled batches of five samples. Readings were performed by three subsequent observers, of whom 
the one who performed the test procedure invariably was the first. Observers were blinded to the results of microscopy and to each others' readings. Readings were carried out at daylight assisted by a standard electricity bulb, between 20 and 30 minutes after application of the sample and buffer. The results of the readings considered were based on consensus agreement, which means that the same result was observed by at least two out of three different observers. Where there was no consensus (in rare cases for the FK60), results of the first observer were considered. To assess inter-observer agreement, results of positive and negative readings as well as line intensity readings were considered. To assess test reproducibility, a panel of six positive samples for $P$. falciparum with various parasite densities $(116,200,1,123,2,900,138,000$ and $275,000 / \mu \mathrm{l}$ ) was tested on five occasions.

\section{Statistical analysis}

For the FK50, true positive results were defined as those with a HRP-2 line visible in samples with $P$. falciparum seen at microscopy, and true negative results as those with no HRP-2 line visible in microscopy-negative samples. Incorrect test results included false-negative samples (those with a microscopic diagnosis of $P$. falciparum but no test line visible), false-positive samples (microscopic negative samples showing a HRP-2 line) and species misidenfications (non-falciparum species showing a HRP-2 line). For the FK60, samples infected with $P$. falciparum and the non-falciparum species were considered separately and the control panels included both microscopy negative samples and samples infected by the non-falciparum species and $P$. falciparum respectively, as shown in Tables 1 and 2. Of note is that, for both tests, samples with pure gametocytaemia were included among the positive $P$. falciparum samples. To avoid complex problems of interpretation, microscopically identified mixed infections were considered separately and not included in the calculations of test characteristics.
Sensitivity and specificity were calculated with 95\% confidence intervals (C.I.) and differences were tested for significance using the chi-square test or, in case of small sample sizes, a two-tailed Fisher's exact test. A p value < 0.05 was considered as significant. In addition, positive and negative likelihood ratios (LHR+ and LHR-) were calculated. Likelihood ratios provide direct information on the tests power to include (LHR+ > 10) or exclude (LHR$<0.1$ ) a disease without being influenced by its prevalence [3]. Inter-observer agreements were assessed using the kappa statistic for paired observers and percentage agreements for all three observers combined. Associations between line intensity readings and parasite densities were assessed for strength of association with Cramer's $V$ for categorical variables, using interpretative criteria published previously [9].

\section{Analysis of discordant results}

For discordant results (i.e. false-negative and false-positive results and species misidentifications) a species-specific polymerase chain reaction (PCR) was performed. Primers and probes sequences detecting small subunit $18 \mathrm{~S}$ rRNA genes were selected according to Rougemont et al [10].

\section{Ethical review}

The study was reviewed and approved by the Institutional Review Board of ITM and by the Ethical Committee of Antwerp University, Belgium.

\section{Results}

\section{SD FK50 Malaria Ag P. falciparum test}

\section{Sample collection}

A total of 452 samples were assessed, including those infected with $P$. falciparum $(\mathrm{n}=324), P$. vivax $(\mathrm{n}=12), P$. ovale $(\mathrm{n}=11), P$. malariae $(\mathrm{n}=6)$, mixed species $(\mathrm{n}=4)$ and negative samples $(\mathrm{n}=95)$. The majority $(272 / 324$, $84.0 \%$ ) of $P$. falciparum samples had been acquired in subSaharan Africa.

\section{Table I: Interpretation of test results of the FK60 for the detection of $P$. falciparum infection}

\begin{tabular}{|c|c|c|}
\hline \multirow[b]{2}{*}{ Test line(s) visible } & \multicolumn{2}{|c|}{ Microscopy result } \\
\hline & $\begin{array}{c}\text { P. falciparum } \\
\text { (asexual and/or gametocytes) }\end{array}$ & $\begin{array}{l}P . \text { vivax, } P \text {. ovale or } P \text {. malariae or } \\
\text { no parasites seen }\end{array}$ \\
\hline \multirow[t]{2}{*}{$\begin{array}{l}\text { Unique HRP-2 line or } \\
\text { Both HRP-2 + pLDH lines }\end{array}$} & True-positives & $\begin{array}{l}\qquad \text { Incorrect results: } \\
\text { - False positive reactions among "no parasites seen" } \\
\text { - Species misidentifications (non-falciparum species } \\
\text { diagnosed as } P \text {. falciparum) }\end{array}$ \\
\hline & Incorrect results: & \\
\hline $\begin{array}{l}\text { No test line or } \\
\text { Unique pLDH line }\end{array}$ & $\begin{array}{l}\text { - False negative reactions among } P \text {. falciparum samples } \\
\text { - Species misidentification ( } P \text {. falciparum diagnosed as non- } \\
\text { falciparum species) }\end{array}$ & True-negatives \\
\hline
\end{tabular}


Table 2: Interpretation of test results of the FK60 for the detection of Plasmodium non-falciparum infection

\begin{tabular}{|c|c|c|}
\hline \multirow[b]{2}{*}{ Test line(s) visible } & \multicolumn{2}{|c|}{ Microscopy result } \\
\hline & $\begin{array}{c}P . \text { vivax, } P \text {. ovale or } \\
P . \text { malariae }\end{array}$ & $\begin{array}{l}P . \text { falciparum or } \\
\text { no parasites seen }\end{array}$ \\
\hline Unique pLDH line & True-positives & $\begin{array}{l}\qquad \text { Incorrect results: } \\
\text { - False positive reactions among "no parasites seen" } \\
\text { - Species misidentifications ( } P \text {. falciparum diagnosed as } \\
\text { non-falciparum species) }\end{array}$ \\
\hline $\begin{array}{l}\text { No test line or } \\
\text { Unique HRP-2 line or } \\
\text { Both pLDH + HRP-2 lines }\end{array}$ & $\begin{array}{l}\qquad \text { Incorrect results: } \\
\text { - False negative reactions among non-falciparum samples } \\
\text { - Species misidentification (non-falciparum species } \\
\text { diagnosed as } P \text {. falciparum) }\end{array}$ & True-negatives \\
\hline
\end{tabular}

Sensitivity, specificity and likelihood ratios

No invalid test results were observed. Additional file 1 shows the overall and detailed test characteristics matched for parasite densities. Sensitivity increased marginally when samples with pure gametocytaemia were subtracted. Sensitivity was related to parasite density, with values at parasite densities $<100 / \mu \mathrm{l}$ significantly lower compared to those at parasite densities $>100 / \mu \mathrm{l}(78.9 \%$ and respectively $97.6 \%$, p < 0.001). Fifteen out of 21 false-negative samples had parasite densities $<100 / \mu$ l (including three samples with pure gametocytaemia), the remaining six had parasite densities ranging from 122 to 400/ $\mu$ l. All these infections had been acquired in Africa. Of the four samples with mixed infection, a single one (with $P$. falciparum and $P$. ovale) gave a false-negative result. The overall specificity was $94.4 \%$, with seven samples that were incorrectly identified: a visible test line was observed among four microscopic negative samples and three $P$. malariae samples. The exclusion power was excellent (LHR- < 0.10) except for parasite densities less than $100 / \mu \mathrm{l}$, the inclusion power was also excellent $(\mathrm{LHR}+>10)$.

\section{Line intensity readings}

Table 3 lists the line intensity readings related to parasite density. Line intensity readings were significantly related to parasite densities with a substantial correlation $(V=$ 0.434, $\mathrm{p}<0.001$ ), but there was considerable overlap between categories. Faint or weak line intensities occurred in $98 / 305(32.1 \%)$ true positive results, mostly but not exclusively at low parasite densities and among all seven false positive results and misidentifications.

\section{Inter-observer agreement and reproducibility}

The inter-observer agreement for positive and negative readings was high, with $97.3 \%$ overall agreement between the three observers and kappa values between 0.95-0.98

Table 3: Line intensity consensus readings for the FK50 according to parasite densities

\begin{tabular}{|c|c|c|c|c|c|c|}
\hline \multirow[b]{2}{*}{ Result by microscopy } & \multicolumn{5}{|c|}{ Line intensity readings, numbers of samples* } & \multirow[b]{2}{*}{ Total } \\
\hline & Negative & Faint & Weak & Medium & Strong & \\
\hline \multicolumn{7}{|l|}{ P. falciparum } \\
\hline All samples combined, total & 22 & 4 & 94 & 66 & $|4|$ & 327 \\
\hline Samples with only gametocytes & 3 & I & 8 & 4 & 1 & 17 \\
\hline Asexual parasite density $0-100 / \mu \mid$ & 12 & 2 & 32 & 9 & 2 & 57 \\
\hline Asexual parasite density $|0|-200 / \mu \mid$ & 3 & & 21 & 8 & 3 & 35 \\
\hline Asexual parasite density $201-1000 / \mu l$ & 4 & I & 28 & 28 & 20 & 81 \\
\hline Asexual parasite density $>1000 / \mu \mathrm{l}$ & & & 5 & 17 & 115 & 137 \\
\hline Other species and no parasites seen, total & 118 & 4 & 3 & & & 125 \\
\hline No parasites seen & 91 & 3 & I & & & 95 \\
\hline P. vivax & 13 & & & & & 13 \\
\hline P. ovale & II & & & & & II \\
\hline P. malariae & 3 & I & $2 * *$ & & & 6 \\
\hline
\end{tabular}


for each pair out of three observers. All discordances in line intensity readings were limited to one category of difference (e.g. a line scored as weak by one observer was scored as medium or faint but not as strong by the other observer(s)). For line intensity readings, overall agreement was $86.9 \%$ and kappa values were between 0.86 and 0.90 . Reproducibility testing showed consistent readings by all three observers on all occasions for three out of six samples. For two other samples discrepancies were limited to differences within one category of line intensity and had no impact on final test result. A single sample (with parasite density $1,123 / \mu \mathrm{l}$ ) provided weak, faint and negative readings upon repeat testing.

\section{Analysis of discordant results}

PCR analysis confirmed the discrepant results in favour of the reference microscopy except for a single sample that was microscopically identified as $P$. malariae and while showing a HRP-2 line, this sample was diagnosed by PCR as a mixed $P$. falciparum/P. malariae infection (Additional file 1). Correcting for this result increased the specificity from $94.4 \%$ to $95.2 \%$ (C.I. $89.6 \%-98.0 \%$ ).

\section{SD FK60 Malaria Ag P. falciparum/Pan test}

\section{Sample collection}

Compared to the panel for the FK50, the numbers of samples with non-falciparum species were increased up to 80 for $P$. vivax and $P$. ovale each and 31 for $P$. malariae, resulting in a total of 614 samples.

\section{Sensitivity, specificity and likelihood ratios}

No invalid test results were recorded. A total of 303 out of $324 P$. falciparum samples were correctly identified. The remaining 21 samples showed no test line. Among the control samples, there was one out of 95 microscopy negative samples that generated a HRP-2 line, and there were six out of 191 non-falciparum samples showing crossreaction with HRP-2 (one sample each with $P$. ovale and $P$. vivax and four samples with $P$. malariae). The resulting test characteristics are displayed in Additional file 2, with an overall sensitivity and a specificity of $94.1 \%$ and $97.6 \%$ respectively. As for the FK50, sensitivities were lower at decreasing parasite densities with significant difference below and above $100 / \mu \mathrm{l}(\mathrm{p}<0.001)$.

For the $P$. non-falciparum species, there were 145 out of 191 samples correctly identified (unique pLDH line visible), six samples had a visible HRP-2 line and 40 samples showed no test line. The resulting overall sensitivities were $87.5 \%, 76.3 \%$ and $45.2 \%$ for $P$. vivax, $P$. ovale and $P$. malariae respectively (Additional file 3 ). Sensitivity for $P$. malariae was significantly lower compared to the two other species $(\mathrm{p}<0.001)$. As for $P$. falciparum, sensitivities declined at lower parasite densities, and dipped at parasite

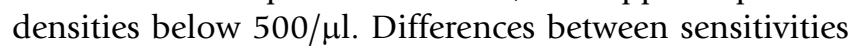

in samples above and below parasite densities of $500 / \mu \mathrm{l}$ reached statistical significance in the case of $P$. ovale $(\mathrm{p}<$ 0.05). Among the microscopic negative samples, there were no false-positive pLDH readings neither were there $P$. falciparum samples that generated a unique pLDH line. The specificity for non-falciparum species was $100 \%$, resulting in high LHR+ values.

Of the four samples with mixed infection, a single one (with $P$. falciparum and $P$. ovale at a parasite density of $700 / \mu \mathrm{l}$ ) showed only a pLDH line, the other mixed infections were correctly identified.

\section{Line intensity readings}

As for the FK50, faint and weak line intensities for the HRP-2 line occurred mostly but not exclusively at low parasite densities. The pLDH line performed worse in terms of visibility, with $224 / 353$ (63.4\%) of true positive readings in the faint or weak categories, as opposed to 90/305 $(29.5 \%)$ for the HRP-2 line ( $\mathrm{p}<0.001)$. The pLDH line showed lowest intensities among the non-falciparum samples, with only 28 out of $80 P$. vivax samples, 5 of 80 $P$. ovale samples and none of the P. malariae samples showing medium or strong line intensities. Line intensity readings for HRP-2 and for $\mathrm{pLDH}$ were related to parasite densities (HRP-2: $V=0.387$, p < 0.05; pLDH: $V=0.457$, p $<0.05$ ) but there was considerable overlap between the different categories. Interestingly, in the case of $P$. falciparum infection, the unique presence of a HRP-2 line pointed almost exclusively (98.1\%, 103/105 of samples) to a parasite density below $1,000 / \mu \mathrm{l}$ (Table 4 ). In addition, the presence of medium or strong pLDH line intensity was invariably associated with parasite densities exceeding $1,000 / \mu \mathrm{l}$ in the case of $P$. falciparum and $500 / \mu \mathrm{l}$ in the case of the non-falciparum species, except for one $P$. falciparum sample with pure gametocytaemia $(12,700 / \mu \mathrm{l})$. Of interest, among the $17 P$. falciparum samples with pure gametocytaemia, there were 14 with HRP-2 lines visible compared to six with pLDH lines visible. HRP-2 lines in samples with non-falciparum species gave faint or weak line intensities except for a single $P$. malariae sample with strong line intensity. This latter sample however proved to be a mixed infection with $P$. falciparum/P. malariae upon PCR analysis (see below).

\section{Inter-observer agreement and reproducibility}

Both target lines performed well for inter-observer agreements, although the results of the HRP-2 line were better than the pLDH line (Table 5). Most discordances in line intensity readings occurred within one category of difference $(81 / 85(95.3 \%)$ and $125 / 129(96.9 \%)$ for HRP-2 and pLDH respectively). The results for the reproducibility testing for the HRP-2 line were comparable to those obtained in the FK50 test. For the pLDH line, consistent readings were recorded by all three observers on all occa- 
Table 4: Reactivity of test lines for the FK60 for $P$. falciparum samples in relation to parasite density

\begin{tabular}{|c|c|c|c|c|}
\hline \multirow[b]{2}{*}{ Parasite densities for $P$. falciparum samples } & \multicolumn{3}{|c|}{ SD FK 60 result: test lines visible } & \multirow[t]{2}{*}{ Total } \\
\hline & None & Unique HRP-2 line & Both HRP-2 and pLDH lines & \\
\hline Pure gametocytaemia & 3 & 8 & 6 & 17 \\
\hline $0-100 / \mu \mid$ & 12 & 42 & 3 & 57 \\
\hline $101-200 / \mu \mid$ & 3 & 18 & 14 & 36 \\
\hline $201-1000 / \mu \mathrm{l}$ & 3 & 35 & 42 & 80 \\
\hline$>1000 / \mu l$ & 0 & 2 & 133 & 135 \\
\hline Total & 21 & 105 & 198 & 324 \\
\hline
\end{tabular}

sions for four out of six samples. Two other samples (with parasite density $116 / \mu \mathrm{l}$ and $200 / \mu \mathrm{l}$ ) provided weak, faint and negative readings upon repeat testing.

\section{Analysis of discordant results}

PCR analysis of all discordances resulted in the following corrections: two samples that were microscopically diagnosed as $P$. ovale and that did not show any test line proved to be mixed $P$. falciparum $/ P$. ovale infections, and one $P$. malariae sample that showed cross-reaction with the HRP-2 line proved to be a mixed P. falciparum $/ P$. malariae infection. When correcting for these results, specificity for the detection of $P$. falciparum and sensitivity for the diagnosis of $P$. malariae increased slightly (Additional files 2 and 3 ).

\section{Side by side comparison of the SD FK50 and SD FK60} assays

A total of 324 P. falciparum samples were assessed by both the FK50 and FK60 tests. Two of them provided different results: one was uniquely positive by FK50 and the other by FK60. Both samples had parasite densities below 100/ $\mu \mathrm{l}$, and displayed faint and weak HRP-2 line intensity readings without a visible $\mathrm{pLDH}$ line. Since both samples belonged to the same parasite density category $(<100 / \mu \mathrm{l})$, data on sensitivity for $P$. falciparum were identical for both assays. In addition to the three $P$. malariae samples that showed HRP-2 lines with the FK50 test, the FK60 showed HRP-2 lines for three other samples, one sample of $P$. malariae, $P$. vivax and $P$. ovale respectively. Among microscopic negative samples, a single sample gave a false-positive HRP-2 line by the FK60 as opposed to four samples for the FK50, resulting in slightly better specificity and $\mathrm{LHR}+$ for the FK60 as compared to the FK50. Agreement between both tests for positive and negative readings and line intensities was excellent (kappa value 0.95) and substantial (kappa value 0.68 ) respectively.

\section{Discussion}

In this study, the performance of two one-step malaria rapid diagnostic tests, the SD FK50 Malaria Ag P. falciparum test (a two-band HRP-2 test) and the SD FK60 Malaria Ag P. falciparum/Pan test (a three-band HRP-2 and pLDH test) was evaluated on large panels of stored samples obtained from international travellers. For both tests, overall sensitivity for the detection of $P$. falciparum was $93.5 \%$, reaching $97.6 \%$ and $100 \%$ at parasite densities above 100 and 1,000/ $\mu$ l respectively. Overall sensitivities for $P$. vivax, $P$. ovale and $P$. malariae for the FK60 test were $87.5 \%, 76.3 \%$ and $45.2 \%$, but they reached $92.6 \%$ and $90.5 \%$ for $P$. vivax and $P$. ovale at parasite densities $>500 /$ $\mu$ l. Specificities were above $95 \%$ for all species. Interobserver agreement and reproducibility were high for both tests.

One of the limitations of the present study was its retrospective design, which made it difficult to trace back causes of discordant results such as previous therapy and

Table 5: Overall agreement and inter-observer agreement between pairs of observers for the FK60 assay

\begin{tabular}{|c|c|c|}
\hline & $\begin{array}{l}\text { Overall agreement between three observers } \\
\text { (\%) }\end{array}$ & $\begin{array}{c}\text { Agreement between pairs of observers } \\
\text { (kappa values) }\end{array}$ \\
\hline \multicolumn{3}{|c|}{ Results expressed as positive and negative readings } \\
\hline HRP-2 & 96.9 & $0.96,0.95,0.96$ \\
\hline $\mathrm{pLDH}$ & 91.9 & $0.88,0.88,0.91$ \\
\hline \multicolumn{3}{|c|}{ Results expressed as line intensity readings } \\
\hline HRP-2 & 86.2 & $0.86,0.86,0.87$ \\
\hline $\mathrm{pLDH}$ & 79.0 & $0.79,0.80,0.80$ \\
\hline
\end{tabular}


the presence of interfering factors (e.g. rheumatoid factor). Further, the study population was not completely homogenous and the small numbers of semi-immune immigrants (who may tolerate low-level parasite densities [5]) were not identified. Another possible limitation is the fact that stored blood samples were used for analysis. Although, on theoretical grounds, there have been concerns about the stability of the target antigens under these conditions [11], previous evaluations of RDTs have been performed on stored samples $[12,13]$ and a prospective evaluation of fresh and stored samples revealed similar results in case of the HRP-2 antigen [14]. In the present study, no obvious differences in test performance were found for samples stored for several ( $>2$ ) years compared to those stored for a shorter period (results not shown), and the samples had not been exposed to repeat thawing and freezing. Further, it should be realised that the present study design did not consider the performance of these RDTs when applied in clinical diagnosis by laboratory technicians in non-endemic settings, who have few exposure to malaria positive samples $[1,2]$. Assessing samples with different parasite densities should be part of the laboratory validation when introducing RDTs in clinical practice, for instance, to train the occasional reader to interpret faint line intensities as positive results. Finally, it should be realized that the present study was performed in a reference setting, with the availability of expert microscopy, trained observers and optimal environmental conditions. Likewise, a calibrated transfer pipette was used instead of the manufacturer's transfer device, in order to ensure correct volume transfer [11]. However, an evaluation of such a test in a reference setting is a logic first step preceding in-depth evaluations and field trials [11].

For both tests and the diagnosis of $P$. falciparum, the sensitivities were in line with those reported in other HRP-2 tests in returned travellers, with sensitivities ranging from $80 \%$ to $99 \%$, depending on the setting and parasite densities [3,15-22]. However, most of these studies, in particular the systematic reviews, addressed the multistep RDTs that are available on the market for a long time $[3,5,6,23]$. By contrast, evaluations of most of the other RDTs displayed on the WHO website are pending [7]. For the nonfalciparum species, the reported sensitivities vary, with decreasing sensitivities for $P$. vivax followed by either $P$. ovale or $P$. malariae $[3,4,15,19,24]$. In a recent meta-analysis on malaria RDTs in international travellers [3], sensitivities for $P$. ovale and $P$. malariae ranging from $36-95 \%$ were mentioned. Part of this wide range in sensitivities is probably due to low sample sizes in different studies. The sample sizes in the present study enabled us to calculate test characteristics with narrower confidence intervals, and consequently this study demonstrates a significantly lower sensitivity for detection of $P$. malariae, as compared to $P$. ovale and $P$. vivax, even at parasite densities above
$500 / \mu$ l. Considering the present methods, two other remarks are to be made. First, in the case of $P$. falciparum, samples with only gametocytes were considered as part of the microscopic positive samples. From the standpoint of travel medicine, this is a recommended choice, but gametocytes may be present even after successful eradication of the asexual forms $[3,5]$. Moving these pure gametocytaemia samples to the "non-malaria" category in the present study collection would add slightly to the sensitivity and the LHR- of both tests (Additional files 1 and 2), at a considerable cost of specificity $(87.0 \%$ and $93.3 \%$ for FK50 and FK60 respectively), but with LHR+ still above 10 . Second, among our control panel not only microscopy negative samples were included, but also samples from other Plasmodium species and we consequently scored species misidentifications as incorrect diagnosis. One could argue that species misidentification can be tolerated as long as the diagnosis of malaria is not missed. Competent malaria diagnosis however requires distinction between at least $P$. falciparum and the other species, as prognosis, therapy, follow-up and epidemiology are different. With regard to both tests, it is reassuring that among the present samples, $P$. falciparum was not erroneously misidentified as a nonfalciparum species (with the exception of a single failure of HRP-2 reactivity in a mixed $P$. falciparum/P. ovale infection), and that misidentification only occurred in the other direction, i.e. non-falciparum species (especially $P$. malariae) were misidentified as $P$. falciparum. The impact of adding other species to the control group however was low in terms of test characteristics: limiting the control panel to exclusively the negative samples would result in a slight increase in specificity $(95.8 \%$ and $98.9 \%$ for the FK50 and FK60 in case of $P$. falciparum respectively), a slight increase in sensitivity for $P$. vivax and $P$. ovale ( $88.8 \%$ and $77.5 \%$ respectively) and a moderate increase in sensitivity for $P$. malariae (58.1\%). Of note are the false positive reactions for the HRP-2 line among the non-falciparum species, in particular among $10 \%$ of our $P$. malariae samples. HRP-2 cross-reaction have been reported for $P$. vivax and $P$. malariae, but not for $P$. ovale $[25,26]$.

For all species, declining sensitivities at lower parasite densities were observed. For P. falciparum this is a wellknown phenomenon $[3-6,11]$. The present study demonstrated this decline for the non-falciparum species as well, indicating a breakpoint at 500/ $\mu \mathrm{l}$. In line with the results from the meta-analysis mentioned above [3], most of the $P$. falciparum false-negative results in this study occurred in samples with parasite densities $<100 / \mu \mathrm{l}$. Although the failure to detect high parasite densities, is also mentioned as a pitfall of malaria RDTs [3-6], no cases of false-negative results were presently found at parasite densities above $400 / \mu \mathrm{l}$. It should be noted however that false-negative results at elevated parasite densities are rare events that await prospective surveillance by incident reporting. 
Further, the results did not show any relation between geographic distribution and false-negative results by HRP2 due to possible genetic variations in HRP-2 target [27], but the majority of samples were acquired in Africa, and more samples should be tested from the Asia-Pacific to rule out an influence of such variations.

In contrast to most other studies, reproducibility and inter-observer agreements of both tests were presently assessed. Line intensity readings (and consequently test results) showed high inter-observer agreements and were also reproducible upon repeat testing, but performances were clearly better for the HRP-2 line as compared to the pLDH line. For the latter line, the preponderance of weak and faint readings for the non-falciparum species is of concern. Furthermore, for the detection of $P$. falciparum, the three-band FK60 test performed as well as the twoband FK50. This is of note, as one could expect the threeband test, which has to meet two optimums of antigenantibody interactions, would perform somewhat less than the two-band test. Although the present devices are not designed to use line intensities as a tool for grading parasite densities, this study also explored their possible diagnostic value. In line with other findings, line intensities were related to parasite density $[17,26,28]$ but considerable overlaps precluded their use as a semi-quantitative estimation of parasite density. However, the FK60 test provided interesting clues to parasite densities below or above $1,000 / \mu \mathrm{l}$ for $P$. falciparum (the unique presence of a HRP-2 line and the presence of medium or strong pLDH line respectively). A similar approach has been described for a HRP-2 and aldolase three-band RDT, for which coreactivity of both test lines pointed to parasite densities of $=40,000 / \mu \mathrm{l}$ [29]. Further product research might refine and expand these possibilities, thereby enlarging the scope of malaria RDTs application [6].

\section{Conclusion}

Taking into account their sensitivity and specificity, interobserver agreement and reproducibility, it is clear that the FK50 and the FK60 tests devices are a valuable adjunct to microscopy for the diagnosis of malaria in a non-endemic setting. They share the limitations of other malaria rapid diagnostic tests, in particular the limited exclusion power for $P$. falciparum malaria at low parasite densities and a lower sensitivity for the non-falciparum species, especially P. malariae. Possible test improvements - apart from the sensitivity - would be an increase in intensity of the pLDH line, and the exploration of the semi-quantitative estimation of the parasite densities.

\section{Abbreviations}

SD FK50: SD FK50 Malaria Ag P. falciparum test (Standard Diagnostics): a two-band "one-step" malaria rapid diag- nostic test for the detection of $P$. falciparum, targeting HRP-2 antigen; SD FK60: SD FK60 Malaria Ag P. falciparum/Pan test (Standard Diagnostics): a three-band "one-step" malaria rapid diagnostic test for the detection of $P$. falciparum and $P$. non-falciparum species, targeting HRP-2 and pLDH antigen; Ag: Antigen; P.: Plasmodium; FHML: Faculty of Health Medicine and Life Sciences, Maastricht, The Netherlands; ITM: Institute of Tropical Medicine, Antwerp, Belgium; PCR: Polymerase Chain Reaction; RDT(s): Rapid Diagnostic Test(s); HRP-2: Histidine Rich Protein-2; pLDH: Parasite lactate dehydrogenase; EDTA: Ethylene diamine tetra-acetic acid; WBC: White Blood Cells; C.I.: Confidence interval; LHR+: Positive likelihood ratio; LHR-: Negative likelihood ratio; rRNA: Ribosomal ribonucleic acid; $\infty$ : Infinite;

\section{Competing interests}

The authors declare that they have no competing interests.

\section{Authors' contributions}

MvdP, PG and JJ designed the study protocol. MvE and EB organized prospective sample collection. MvdP and PG carried out the test evaluations, LC performed PCR analysis. MvdP, PG and JJ analyzed and interpreted the results and drafted the manuscript, PG performed statistical analysis. All authors contributed to the discussion of the results and the redaction of the manuscript, they all approved the final manuscript.

\section{Additional material}

\section{Additional file 1}

Test characteristics of the FK50 related to parasite densities. Click here for file [http://www.biomedcentral.com/content/supplementary/14752875-8-90-S1.doc]

\section{Additional file 2}

Test characteristics of the FK60 for P. falciparum samples according to parasite densities.

Click here for file

[http://www.biomedcentral.com/content/supplementary/14752875-8-90-S2.doc]

\section{Additional file 3}

Test characteristics of the FK60 for non-falciparum species according to parasite densities.

Click here for file

[http://www.biomedcentral.com/content/supplementary/1475-

2875-8-90-S3.doc]

\section{Acknowledgements}

We would like to thank Veronique Guns and the staff of the Central Laboratory of Clinical Biology for technical support. 


\section{References}

I. Hänscheid T: Current strategies to avoid misdiagnosis of malaria. Clin Microbiol Infect 2003, 9:497-504.

2. Wiese L, Bruun B, Baek L, Friis-Moller A, Gahrn-Hansen B, Hansen J, Heltberg O, Hojbjerg T, Hornstrup MK, Kvinesdal B, Gomme G, Kurtzhals JA: Bedside diagnosis of imported malaria using the Binax Now malaria antigen detection test. Scand J Infect Dis 2006, 38:1063-1068.

3. Marx A, Pewsner D, Egger M, Nuesch R, Bucher HC, Genton B, Hatz $C$, Juni P: Meta-analysis: accuracy of rapid tests for malaria in travelers returning from endemic areas. Ann Intern Med 2005, I 42:836-846.

4. Moody AH, Chiodini PL: Non-microscopic method for malaria diagnosis using OptiMAL IT, a second-generation dipstick for malaria pLDH antigen detection. Br J Biomed Sci 2002, 59:228-231.

5. Murray CK, Gasser RA Jr, Magill AJ, Miller RS: Update on Rapid Diagnostic Testing for Malaria. Clin Microbiol Rev 2008, 21:97-II0.

6. Wongsrichanalai C, Barcus MJ, Muth S, Sutamihardja A, Wernsdorfer WH: A review of malaria diagnostic tools: microscopy and rapid diagnostic test (RDT). Am J Trop Med Hyg 2007, 77:I19-127.

7. World Health Organization: List of known commercially-available antigen-detecting malaria RDTs: information for national public health services and UN Agencies wishing to procure RDTs. [http://www.wpro.who.int/NR/rdonlyres/ 9C624A45-3554-4695-8CII-233DB743FC7A/0/ MD table23 ISOI3/485criteriarev04I207.pdf].

8. Bell DR, Wilson DW, Martin LB: False-positive results of a Plasmodium falciparum histidine-rich protein 2-detecting malaria rapid diagnostic test due to high sensitivity in a community with fluctuating low parasite density. Am J Trop Med Hyg 2005, 73:199-203.

9. Stamatopoulos B, Meuleman N, Haibe-Kains B, Duvillier H, Massy M, Martiat P, Bron D, Lagneaux L: Quantification of ZAP70 mRNA in $B$ cells by real-time $P C R$ is a powerful prognostic factor in chronic lymphocytic leukemia. Clin Chem 2007, 53: I757-I 766.

10. Rougemont M, Van Saanen M, Sahli R, Hinrikson HP, Bille J, Jaton K: Detection of four Plasmodium species in blood from humans by I 8S rRNA gene subunit-based and species-specific realtime PCR assays. J Clin Microbiol 2004, 42:5636-5643.

II. Bell D, Peeling RW: Evaluation of rapid diagnostic tests: malaria. Nat Rev Microbiol 2006, 4:S34-S38.

12. Craig MH, Bredenkamp BL, Williams CH, Rossouw EJ, Kelly VJ, Kleinschmidt I, Martineau A, Henry GF: Field and laboratory comparative evaluation of ten rapid malaria diagnostic tests. Trans $R$ Soc Trop Med Hyg 2002, 96:258-265.

13. Piper R, Lebras ], Wentworth L, Hunt-Cooke A, Houze S, Chiodini P, Makler M: Immunocapture diagnostic assays for malaria using Plasmodium lactate dehydrogenase (pLDH). Am J Trop Med Hyg 1999, 60:109-118.

14. Mayxay M, Pukrittayakamee S, Chotivanich K, Looareesuwan S, White NJ: Persistence of Plasmodium falciparum HRP-2 in successfully treated acute falciparum malaria. Trans $R$ Soc Trop Med Hyg 200I, 95:179-182.

15. De Monbrison F, Gerome P, Chaulet JF, Wallon M, Picot S, Peyron F: Comparative diagnostic performance of two commercial rapid tests for malaria in a non-endemic area. Eur J Clin Microbiol Infect Dis 2004, 23:784-786.

16. Durand F, Crassous B, Fricker-Hidalgo H, Carpentier F, Brion JP, Grillot R, Pelloux H: Performance of the Now Malaria rapid diagnostic test with returned travellers: a 2-year retrospective study in a French teaching hospital. Clin Microbiol Infect 2005, I I:903-907.

17. Farcas GA, Zhong KJ, Lovegrove FE, Graham CM, Kain KC: Evaluation of the Binax NOW ${ }^{\circledR}$ ICT test versus polymerase chain reaction and microscopy for the detection of malaria in returned travelers. Am J Trop Med Hyg 2003, 69:589-592.

18. Gatti S, Gramegna M, Bisoffi Z, Raglio A, Gulletta M, Klersy C, Bruno A, Maserati R, Madama S, Scaglia M: A comparison of three diagnostic techniques for malaria: a rapid diagnostic test (NOW Malaria), PCR and microscopy. Ann Trop Med Parasitol 2007, I 0I:195-204.

19. Grobusch MP, Hanscheid T, Zoller T, Jelinek T, Burchard GD: Rapid immunochromatographic malarial antigen detection unreli- able for detecting Plasmodium malariae and Plasmodium ovale. Eur J Clin Microbiol Infect Dis 2002, 21:818-820.

20. Grobusch MP, Hanscheid T, Gobels K, Slevogt H, Zoller T, Rogler G, Teichmann D: Comparison of three antigen detection tests for diagnosis and follow-up of falciparum malaria in travellers returning to Berlin, Germany. Parasitol Res 2003, 89:354-357.

21. Jelinek T, Grobusch MP, Schwenke S, Steidl S, von Sonnenburg F, Nothdurft HD, Klein E, Loscher T: Sensitivity and specificity of dipstick tests for rapid diagnosis of malaria in nonimmune travelers. J Clin Microbiol 1999, 37:721-723.

22. Ende J Van den, Vervoort T, Van Gompel A, Lynen L: Evaluation of two tests based on the detection of histidine rich protein 2 for the diagnosis of imported Plasmodium falciparum malaria. Trans R Soc Trop Med Hyg 1998, 92:285-288.

23. Cruciani M, Nardi S, Malena M, Bosco O, Serpelloni G, Mengoli C: Systematic review of the accuracy of the Para Sight ${ }^{\mathrm{TM}}-\mathrm{F}$ test in the diagnosis of Plasmodium falciparum malaria. Med Sci Monit 2004, I0:MT8I-MT88.

24. Playford EG, Walker J: Evaluation of the ICT Malaria P.f/P.v and the OptiMal rapid diagnostic tests for malaria in febrile returned travellers. J Clin Microbiol 2002, 40:4I66-4I7I.

25. lqbal J, Hira PR, Sher A, Al Enezi AA: Diagnosis of imported malaria by Plasmodium lactate dehydrogenase (pLDH) and histidine-rich protein 2 (PfHRP-2)-based immunocapture assays. Am J Trop Med Hyg 200I, 64:20-23.

26. Pieroni P, Mills CD, Ohrt C, Harrington MA, Kain KC: Comparison of the Para Sight ${ }^{\mathrm{TM}}-\mathrm{F}$ test and the ICT Malaria Pf ${ }^{\mathrm{TM}}$ test with the polymerase chain reaction for the diagnosis of Plasmodium falciparum malaria in travellers. Trans $\mathrm{R}$ Soc Trop Med Hyg 1998, 92:166-169.

27. Baker J, McCarthy J, Gatton M, Kyle DE, Belizario V, Luchavez J, Bell D, Cheng Q: Genetic diversity of Plasmodium falciparum Histidine-Rich Protein 2 (PfHRP2) and its effect on the performance of PfHRP2-Based rapid diagnostic tests. J Infect Dis 2005, 192:870-877.

28. Mason DP, Kawamoto F, Lin K, Laoboonchai A, Wongsrichanalai C: A comparison of two rapid field immunochromatographic tests to expert microscopy in the diagnosis of malaria. Acta Trop 2002, 82:51-59.

29. Richter J, Gobels K, Muller-Stover I, Hoppenheit B, Haussinger D: Co-reactivity of plasmodial histidine-rich protein 2 and aldolase on a combined immuno-chromographic-malaria dipstick (ICT) as a potential semi-quantitative marker of high Plasmodium falciparum parasitaemia. Parasitol Res 2004, 94:384-385

\section{Publish with Bio Med Central and every scientist can read your work free of charge}

"BioMed Central will be the most significant development for disseminating the results of biomedical research in our lifetime. "

Sir Paul Nurse, Cancer Research UK

Your research papers will be:

- available free of charge to the entire biomedical community

- peer reviewed and published immediately upon acceptance

- cited in PubMed and archived on PubMed Central

- yours - you keep the copyright

Submit your manuscript here:

http://www.biomedcentral.com/info/publishing_adv.asp
BioMedcentral 